\title{
Retrospective analysis of capecitabine and oxaliplatin (XELOX) plus bevacizumab as a first-line treatment for Japanese patients with metastatic colorectal cancer
}

\author{
YASUTAKE UCHIMA, TAKAFUMI NISHII, YASUHITO ISEKI, MARIKO ISHII, SOICHIRO HIRAMATSU, \\ TAKEHIKO IWAUCHI, JUNYA MORIMOTO, KINSHI KOSAKA, SEIKA TEI and KAZUHIRO TAKEUCHI
}

Department of Surgery, Fuchu Hospital, Izumi, Osaka 5940076, Japan

Received June 7, 2013; Accepted September 11, 2013

DOI: $10.3892 / \mathrm{mco} .2013 .205$

\begin{abstract}
XELOX plus bevacizumab is an effective treatment strategy and has a manageable tolerability profile when administered to Japanese patients with metastatic colorectal cancer (mCRC). In this study, we retrospectively reviewed cases in which XELOX plus bevacizumab were administered in order to evaluate its efficacy and safety in clinical practice. In total, 40 patients with mCRC who presented at Fuchu Hospital received XELOX plus bevacizumab as a first-line treatment between September, 2009 and April, 2012. Eligible patients had histologically confirmed mCRC. XELOX consisted of a 2-h intravenous infusion of oxaliplatin $130 \mathrm{mg} / \mathrm{m}^{2}$ on day 1 plus oral capecitabine $1,000 \mathrm{mg} / \mathrm{m}^{2}$ twice daily for 2 weeks of a 3-week cycle. Overall survival (OS) and survival benefit were analyzed when patients continued with XELOX plus bevacizumab beyond disease progression. The median progression-free survival (PFS) was 290 days [95\% confidence interval (CI): 222-409 days] and the median OS was 816 days (95\% CI: 490 days-not calculated). The response rate (RR; complete plus partial response) was $67.5 \%$, and the disease control rate (RR plus stable disease) was $90 \%$. The most common adverse events observed following administration of XELOX plus bevacizumab were neurosensory toxicity $(82.5 \%)$, anorexia (50\%), hypertension (45\%) and a decrease in the platelet count (40\%). The most common grade $3 / 4$ adverse events were neurosensory toxicity (15\%) and fatigue $(15 \%)$. In conclusion, XELOX plus bevacizumab may be considered a routine first-line treatment option for patients with mCRC. Notably, the combination of capecitabine and bevacizumab was safe with an acceptable toxicity profile and induced a significant rate of disease control.
\end{abstract}

Correspondence to: Dr Yasutake Uchima, Department of Surgery, Fuchu Hospital, 1-10-17 Hiko-cho, Izumi, Osaka 5940076, Japan E-mail: y_uchima@seichokai.or.jp

Key words: capecitabine, oxaliplatin, bevacizumab, colorectal cancer

\section{Introduction}

Colorectal cancer is the third most common type of cancer worldwide, with one million new cases diagnosed annually (1). In Japan, colorectal cancer is the second most common type of cancer and the third most common cause of mortality (2).

FOLFOX4, a bi-weekly schedule of intravenous bolus and infusion of 5-fluorouracil/folinic acid (5-FU/FA) plus oxaliplatin $\left(\right.$ Elplat $\left.^{\circledR}\right)$, is a widely used regimen for the first-line treatment of metastatic colorectal cancer (mCRC) $(3,4)$. However, oral fluoropyrimidines can replace the intravenous fluoropyrimidine component of combination regimens. Capecitabine (Xeloda ${ }^{\circledR}$ ) is an oral fluoropyrimidine with similar efficacy to bolus 5-FU/ FA in the first-line treatment of $\mathrm{mCRC}$ and as adjuvant therapy for stage III colon cancer (5-7). The efficacy of capecitabine and a 3-week dose of oxaliplatin (XELOX regimen) has also been demonstrated to be inferior to 5-FU/FA plus oxaliplatin (FOLFOX4 or FOLFOX6) in the first- and second-line treatment of patients with mCRC (8-10). The addition of bevacizumab (Avastin ${ }^{\circledR}$ ) to oxaliplatin-based chemotherapy significantly improved progression-free survival (PFS) by $20 \%$ in the first-line treatment of mCRC (11). XELOX plus bevacizumab is an effective treatment strategy and has a manageable tolerability profile when administered to Japanese patients with mCRC (12). In this study, we retrospectively reviewed cases in which XELOX plus bevacizumab was administered to evaluate its efficacy and safety in clinical practice.

\section{Patients and methods}

Patients. In total, 40 patients, 22 males and 18 females with a median age of 62.5 years, with mCRC presented at the Fuchu Hospital. These patients were administered XELOX plus bevacizumab as a first-line treatment between September, 2009 and April, 2012. Eligible patients had histologically confirmed mCRC. Other inclusion criteria were an Eastern Cooperative Oncology Group (ECOG) performance status of $0-1$ and adequate hematological, liver and renal functions. Other assessments were carried out at the investigator's discretion.

Treatment. Oxaliplatin was purchased from Yakult Honsha Co., Ltd. (Tokyo, Japan) and capecitabine and bevacizumab 
were purchased from Chugai Pharmaceutical Co., Ltd. (Tokyo, Japan). XELOX consisted of a 2 -h intravenous infusion of oxaliplatin $130 \mathrm{mg} / \mathrm{m}^{2}$ on day 1 plus oral capecitabine $1,000 \mathrm{mg} / \mathrm{m}^{2}$ twice daily for 2 weeks of a 3 -week cycle. The first dose of capecitabine was administered on the evening of day 1 and the last dose was administered on the morning of day 15 . Bevacizumab at a dose of $7.5 \mathrm{mg} / \mathrm{kg}$ was administered as a $30-$ to $90-\mathrm{min}$ intravenous infusion prior to oxaliplatin on day 1 of the 3 -week cycle. Treatment was continued until disease progression, intolerable adverse events or the withdrawal of consent.

Treatment was interrupted if grade 2-4 adverse events occurred. No dose modification of bevacizumab was performed. The dose of capecitabine was adjusted for grade 3 or 4 thrombocytopenia or neutropenia, febrile neutropenia or non-hematological toxicities of grade $\geq 2$, according to the standard scheme described in detail by Doi et al (12). The dose of oxaliplatin was reduced to $100 \mathrm{or} 85 \mathrm{mg} / \mathrm{m}^{2}$ when patients experienced grade 3 or 4 thrombocytopenia or neutropenia, febrile neutropenia or a grade 3 non-hematological toxicity, grade 3 neurosensory toxicity lasting $>7$ days, or grade 2 neurosensory toxicity persisting between cycles. If grade 3 neurosensory toxicity persisted between cycles, oxaliplatin was discontinued. This treatment plan was almost identical to that of the NO16966 study (11).

If oxaliplatin and/or bevacizumab were discontinued, treatment with the remaining components were continued, such as capecitabine with or without bevacizumab subsequent to the discontinuation of oxaliplatin and XELOX or capecitabine after the discontinuation of bevacizumab. The continuation of oxaliplatin or bevacizumab without capecitabine was not permitted.

Evaluation of the methods. Objective tumor responses were evaluated according to the Response Evaluation Criteria in Solid Tumors version 1.0 (RECIST v 1.0) by each attending phycisian. Adverse events were assessed according to the Common Terminology Criteria for Adverse Events version 3.0 (CTCAE v 3.0).

Statistical analysis. Statistical analyses were performed using the Statcel 2 software program (OMS, Saitama, Japan). The evaluation of response and progression was based on measurements reported by the radiologist. Complete and partial response required subsequent confirmation of response following an interval of at least 4 weeks. All clinical courses including subsequent chemotherapy were followed-up until death or last contact.The Kaplan-Meier method was used to evaluate the median duration of treatment, progression-free survival, and overall survival (OS). The median duration of treatment was calculated from the date that treatment was initiated to the date of disease progression or the cessation of treatment for any reason, whichever date occurred first. Progression-free survival was calculated from the date at which the treatement was started to the earlier date of disease progression or death. Without contradictory dates, patients who were lost to follow-up were assumed to have progressed at the last date of confirmation and to be progression-free. For patients whose treatments were ceased without progression and who had received subsequent surgery or an alternative treatment, progression-free survival
Table I. Patient characteristics.

Characteristics

No. of patients

Age (years)
Median (range)
Gender (\%)
Male
Female

$65.2(51-79)$

Eastern Cooperative Oncology

Group performance status

$0(\%)$

Primary tumor site $(\%)$

Colon

Rectum

Stage at first diagnosis (\%)

Local regional

Metastatic

Site of metastasis (\%)

Liver

Lung

Lymph node

Peritoneum

Local recurrence

Line of treatment (\%)

First

Prior adjuvant therapy (\%)

No

Yes

was defined as the time from the initiation of treatment to the date of its cessation. OS was calculated from the date at which treatment was started to death or that of the last contact. Patients who were lost to follow-up were assumed to have succumbed to their disease at the last contact. The cutoff date was April 30, 2013 for progression-free survival and OS.

\section{Results}

Table I shows the characteristics of the 40 enrolled patients. The median age of the patients was 65.2 (range, 51-79 years). Of the 40 patients, 22 were male and 18 were female. The ECOG performance status was 0 in all 40 patients. The most common sites of metastasis were the liver, lungs, lymph nodes and peritoneum.

The median duration of treatment was 170.2 (range, 12-448 days) with a median of 8.0 (range, 1-21 treatment cycles). XELOX plus bevacizumab combination therapy was administered for a median of 6.5 (range, 1-18 cycles). Following the discontinuation of oxaliplatin, 4 patients $(10 \%)$ continued with capecitabine and bevacizumab combination therapy and received a median of 3.2 (range, 1-5 cycles). Five patients (12.5\%) received capecitabine monotherapy for a median of 7.7 (range, 4-12 cycles). One patient 
Table II. Analysis of efficacy.

\begin{tabular}{lll}
\hline A, Endpoint & & \\
\hline Survival & Days & $95 \%$ CI \\
\hline Median progressive-free survival & 290 & $222-409$ \\
Median overall survival & 816 & $490-\mathrm{NC}$ \\
\hline
\end{tabular}

$\mathrm{B}$, Patient response

\begin{tabular}{lc}
\hline Response rate & No. of patients $(\%)$ \\
\hline Complete response & $1(2.5)$ \\
Partial response & $26(65)$ \\
Stable disease & $9(22.5)$ \\
Progressive disease & $1(2.5)$ \\
Not evaluable & $3(7.5)$ \\
\hline
\end{tabular}

NC, not calculated; CI, confidence interval.

Table III. The second and third line regimens used for patients receiving bevacizumab as the first-line treatment.

\begin{tabular}{llc}
\hline Line of treatment & \multicolumn{1}{c}{ Regimen } & $\begin{array}{c}\text { No. of } \\
\text { patients }(\%)\end{array}$ \\
\hline Second line & $\begin{array}{c}\text { Combination with } \\
\text { bevacizumab } \\
\text { Chemotherapy only }\end{array}$ & $7(36.8)$ \\
& $\begin{array}{l}\text { Combination with } \\
\text { cetuximab/panitumumab }\end{array}$ & $8(21.1)$ \\
Third line & $\begin{array}{l}\text { Combination with } \\
\text { bevacizumab }\end{array}$ & $1(16.7)$ \\
& $\begin{array}{l}\text { Chemotherapy only } \\
\text { Combination with }\end{array}$ & $2(33.3)$ \\
& cetuximab/panitumumab & $4(50)$ \\
\hline
\end{tabular}

received XELOX therapy for 2 cycles during the permanent discontinuation of bevacizumab (Fig. 1).

At the final data cut-off date of August 31, 2012, the median duration of follow-up was 500.5 days. Seventeen patients $(42.5 \%)$ succumbed to disease progression and two patients were still receiving medication.

The analysis of efficacy is presented in Table II. The median PFS was 290 [95\% confidence interval (CI): 222-409 days] and the median OS was 816 (95\% CI: 490 days-not calculated). The response rate (RR; complete plus partial response) was $67.5 \%$ and the disease control rate (RR plus stable disease) was $90 \%$.

Table III shows the second- and third-line regimens used for patients treated with bevacizumab in the first-line regimen. Results revealed that $36.8 \%$ of patients who were treated with
Table IV. Incidence of common adverse events.

\begin{tabular}{lcll}
\hline & \multicolumn{1}{c}{ Grade $1-4$} & & Grade $3-4$ \\
\cline { 2 - 2 } Adverse event & No. of patients $(\%)$ & No. of patients $(\%)$ \\
\hline Hypertension & $18(45)$ & & $0(0)$ \\
Neurosensory toxicity & $33(82.5)$ & & $6(15)$ \\
Anorexia & $20(50)$ & & $3(7.5)$ \\
Fatigue & $12(30)$ & $6(15)$ \\
Hand-foot syndrome & $15(37.5)$ & $0(0)$ \\
Nausea/vomitting & $7(17.5)$ & $0(0)$ \\
Diarrhea & $2(5)$ & $0(0)$ \\
Oral ulcer & $7(17.5)$ & $0(0)$ \\
Allergic reaction & $2(5)$ & $2(5)$ \\
Hiccups & $1(2.5)$ & $1(2.5)$ \\
Neutrophil count & $12(30)$ & $0(0)$ \\
decreased & $16(40)$ & $0(0)$ \\
Platelet count & & \\
decreased & &
\end{tabular}

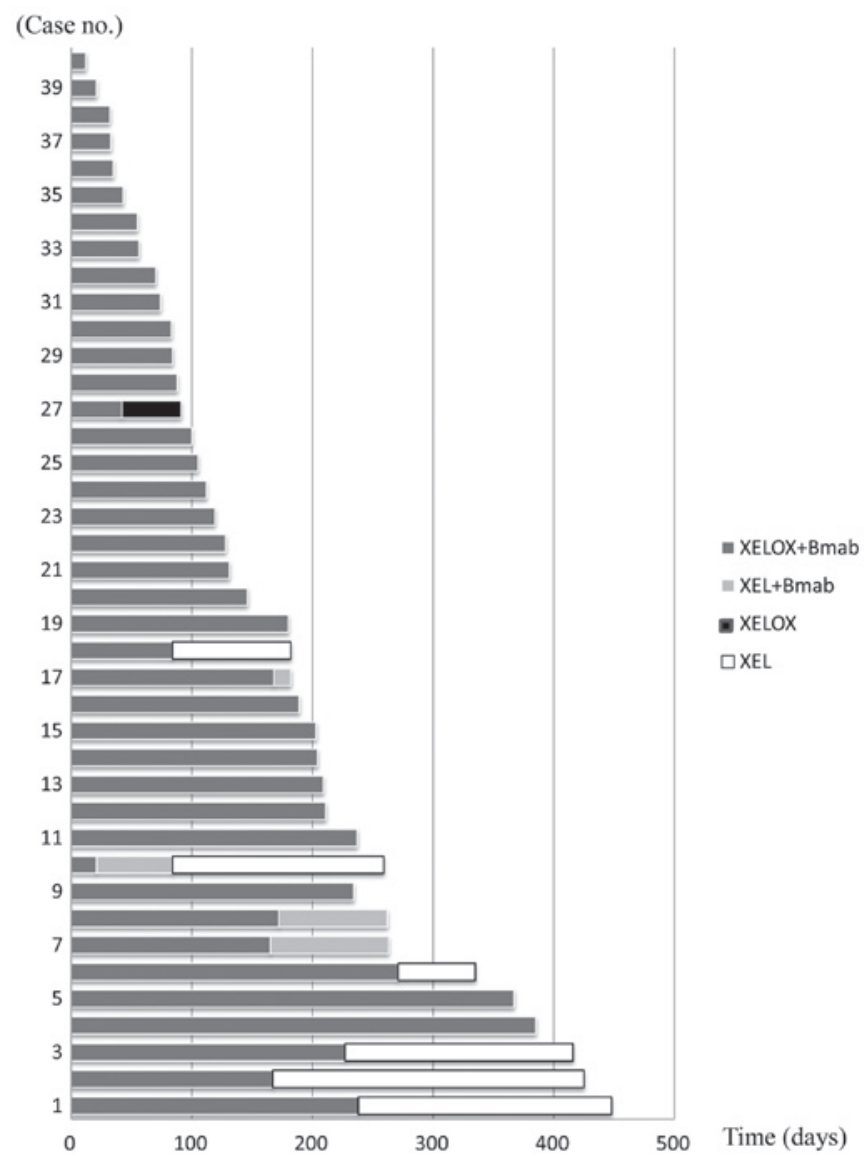

Figure 1. Period of treatment of XEL $(\mathrm{OX}) \pm$ bevacizumab (Bmab).

bevacizumab in the second-line regimen were administered bevacizumab continuously. A total of $42.1 \%$ of patients in the second-line regimen were administered combination chemotherapy with cetuximab or panitumumab. 
The adverse events that occurred in all 40 patients are provided in Table IV. The most common adverse events with XELOX plus bevacizumab were neurosensory toxicity $(82.5 \%)$, anorexia (50\%), hypertension (45\%) and a decrease in the platelet count $(40 \%)$. The most common grade $3 / 4$ adverse events were neurosensory toxicity $(15 \%)$ and fatigue $(15 \%)$. Regarding patients receiving XELOX plus bevacizumab, dose reductions were required for capecitabine in 6 patients $(15 \%)$ due to hand-foot syndrome $(n=5)$ and diarrhea $(n=1)$. Capecitabine doses were reduced to $75 \%$ of the starting dose in all 6 patients. Dose reductions were required for oxaliplatin in 10 patients $(25 \%)$ due to neurosensory toxicity and the oxaliplatin dose was reduced to $100 \mathrm{mg} / \mathrm{m}^{2}$ in all of these patients.

\section{Discussion}

Results of this study have demonstrated the safety and efficacy of XELOX with bevacizumab in combination with oxaliplatin $130 \mathrm{mg} / \mathrm{m}^{2}$ plus oral capecitabine $1,000 \mathrm{mg} / \mathrm{m}^{2}$ in Japanese patients. Of particular significance are our novel results demonstrating the safety and efficacy of the international standard-dose XELOX with bevacizumab in Japanese patients. No fatal adverse events occurred and any complications arising were managed successfully using appropriate support care and drug cessation/dose reductions.

The results of randomized controlled trials in patients with advanced colorectal cancer demonstrated that the median OS was 16-23 months in patients who received bevacizumab with fluoropyrimidine-based chemotherapy, including 5-FU/FA, irinotecan plus 5-FU/leucovorin (IFL), 5-FU/IFL plus oxaliplatin (FOLFOX) and capecitabine plus oxaliplatin (XELOX), as first-line chemotherapy $(11,13,14)$.

Previous randomized or observation trials that included the XELOX plus bevacizumab regimen as first-line therapy have been conducted mainly in North America and Europe $(11,15,16)$. The NO16966 study $(10,15)$ demonstrated a longer PFS and OS in the XELOX plus bevacizumab arm than that with the XELOX plus placebo arm in the subgroup analysis, which reported a median PFS of 9.3 vs. 7.4 months, hazard ratio $(\mathrm{HR})=0.77(95 \% \mathrm{CI}$ : $0.63-0.94, \mathrm{P}=0.0026)$ and a median OS of 21.6 vs. 18.8 months (HR was not shown) (15). Furthermore, another phase III trial (CAIRO2) reported a RR of $50.0 \%$, a median PFS of 10.7 months and a median OS of 20.3 months in the XELOX plus bevacizumab arm (16). A Japanese clinical trial of XELOX plus bevacizumab in patients with mCRC reported that the median OS was 27.4 months and that the median progression-survival was 11.0 months (12). In this study, the median OS was 816 days, the median PFS was 290 days and the RR was $67.5 \%$. These efficacy results are similar to those obtained in first-line therapy with XELOX plus bevacizumab.

The safety profile observed in the present study was similar to that observed in previous clinical trials with Western patients, including the NO16966 study $(11,16,17)$. Notably, the incidence of grade $3 / 4$ diarrhea and nausea/vomiting was $0 \%$, which is markedly lower than that reported with XELOX plus bevacizumab in previous phase II and III studies $(19-21 \%)(11,16,17)$. A lower incidence of diarrhea and nausea/vomiting has been reported in other studies of Japanese or Asian patients treated with fluoropyrimidine-based chemotherapy $(18,19)$. The reason for this regional variation remains unclear; however, it is hypothesized that differences in dietary folate intake is a potential explanation (20). The incidence of all grades of hand-foot syndrome (37.5\%) was similar to that in the XELOX plus bevacizumab arm of the NO16966 study (39\%) (15).

Approximately $90 \%$ of patients with severe peripheral neuropathy reportedly improve 20 weeks following the discontinuation of oxaliplatin (3). The results of the OPTIMOX-1 study demonstrated that a 'stop-and-go' approach employing an oxaliplatin-free interval resulted in decreased neurotoxicity, but did not affect OS in patients receiving FOLFOX as initial therapy for mCRC (21). The antitumor effects of capecitabine used alone on $\mathrm{mCRC}$ have been reported to be similar to those of intravenous 5-FU/IFL therapy (22). Capecitabine and bevacizumab therapy showed a RR of $34 \%$ and median PFS and OS were 10.8 and 18 months, respectively (23). Regarding peripheral neuropathy, the overall incidence of peripheral neuropathy in our study $(82.5 \%)$ was similar to that in the XELOX plus bevacizumab arm of previous studies $(84-93 \%)(12,15)$. In the present study, following the discontinuation of oxaliplatin, 4 patients $(10 \%)$ continued with capecitabine and bevacizumab combination therapy and received a median of 3.2 (range, $1-5$ cycles). Five patients $(12.5 \%)$ received capecitabine monotherapy for a median of 7.7 (range, 4-12 cycles). One patient received XELOX therapy for 2 cycles during the permanent discontinuation of bevacizumab. Peripheral neuropathy disappeared and successful tumor control was achieved (partial response continued).

In conclusion, findings of this study have demonstrated that the survival benefit of XELOX plus bevacizumab in Japanese patients with mCRC was similar to that observed in previous clinical trials from Western countries. Therefore, XELOX plus bevacizumab may be considered a routine first-line treatment option for patients with mCRC. Additionally, the combination of capecitabine and bevacizumab was found to be safe with an acceptable toxicity profile, and induced a significant rate of disease control.

\section{References}

1. Parkin DM, Bray F, Ferlay J, et al: Global cancer statistics, 2002. CA Cancer J Clin 55: 74-108, 2005.

2. Kotake K, Honjo S, Sugihara K, et al: Changes in colorectal cancer during a 20-year period: an extended report from the multi-institutional registry of large bowel cancer, Japan. Dis Colon Rectum 46 (Suppl 10): S32-S43, 2003.

3. de Gramont A, Figer A, Seymour M, et al: Leucovorin and fluorouracil with or without oxaliplatin as first-line treatment in advanced colorectal cancer. J Clin Oncol 18: 2938-2947, 2000.

4. Goldberg RM, Sargent DJ, Morton RF, et al: A randomized controlled trial of fluorouracil plus leucovorin, irinotecan, and oxaliplatin combinations in patients with previously untreated metastatic colorectal cancer. J Clin Oncol 22: 23-30, 2004.

5. Hoff PM, Ansari R, Batist G, et al: Comparison of oral capecitabine versus intravenous fluorouracil plus leucovorin as first-line treatment in 605 patients with metastatic colorectal cancer: results of a randomized phase III study. J Clin Oncol 19: 2282-2292, 2001

6. Van Cutsem E, Twelves C, Cassidy J, et al: Oral capecitabine compared with intravenous fluorouracil plus leucovorin in patients with metastatic colorectal cancer: results of a large phase III study. J Clin Oncol 19: 4097-4106, 2001.

7. Twelves C, Wong A, Nowacki MP, et al: Capecitabine as adjuvant treatment for stage III colon cancer. N Engl J Med 352: 2696-2704, 2005. 
8. Cassidy J, Clarke S, Diaz-Rubio E, et al: Randomized phase III study of capecitabine plus oxaliplatin compared with fluorouracil/folinic acid plus oxaliplatin as first-line therapy for metastatic colorectal cancer. J Clin Oncol 26: 2006-2012, 2008

9. Ducreux M, Bennouna J, Hebbar M, et al: Capecitabine plus oxaliplatin (XELOX) versus 5-fluorouracil/leucovorin plus oxaliplatin (FOLFOX-6) as first-line treatment for metastaic colorectal cancer. Int J Cancer 128: 682-690, 2011.

10. Rothenberg ML, Cox JV, Butts C, et al: Capecitabine plus oxaliplatin (XELOX) versus 5-fluorouracil/folinic acid plus oxaliplatin (FOLFOX-4) as second-line therapy in metastatic colorectal cancer: a randomized phase III noninferiority study. Ann Oncol 19: 1720-1726, 2008.

11. Saltz LB, Clarke S, Diaz-Rubio E, et al: Bevacizumab in combination with oxaliplatin-based chemotherapy as first-line therapy in metastatic colorectal cancer: a randomized phase III study. J Clin Oncol 26: 2013-2019, 2008.

12. Doi T, Boku N, Kato K, et al: Phase I/II study of capecitabine plus oxaliplatin (XELOX) plus bevacizumab as first-line therapy in Japanese patients with metastatic colorectal cancer. Jpn J Clin Oncol 40: 913-920, 2010.

13. Hurwitz H, Fehrenbacher L, Novotny W, et al: Bevacizumab plus irinotecan, fluorouracil, and leucovorin for metastatic colorectal cancer. N Engl J Med 350: 2335-2342, 2004.

14. Kabbinavar FF, Schulz J, McCleod M, et al: Addition of bevacizumab to bolus fluorouracil and leucovorin in first-line metastatic colorectal cancer: results of a randomized phase II trial. J Clin Oncol 23: 3697-3705, 2005.

15. Cassidy J, Clarke S, Diaz-Rubio E, et al: XELOX vs FOLFOX-4 as first-line therapy for metastatic colorectal cancer: NO16966 updated results. Br J Cancer 105: 58-64, 2011.
16. Tol J, Koopman M, Cats A, et al: Chemotherapy, bevacizumab, and cetuximab in metastatic colorectal cancer. N Engl J Med 360: 563-572, 2009

17. Hochster HS, Hart LL, Ramanathan RK, et al: Safety and efficacy of oxaliplatin and fluoropyrimidine regimens with or without bevacizumab as first-line treatment of metastatic colorectal cancer: results of the TREE Study. J Clin Oncol 26: 3523-3539, 2008

18. Hyodo I, Shirao K, Doi T, et al: A phase II study of the global dose and schedule of capecitabine in Japanese patients with metastatic colorectal cancer. Jpn J Clin Oncol 36: 410-417, 2006.

19. Yoshino T, Boku N, Onozawa Y, et al: Efficacy and safety of an irinotecan plus bolus 5-fluorouracil and L-leucovorin regimen for metastatic colorectal cancer in Japanese patients: experience in a single institution in Japan. Jpn J Clin Oncol 37: 686-691, 2007.

20. Haller DG, Cassidy J, Clarke SJ, et al: Potential regional differences for the tolerability profiles of fluoropyrimidines. J Clin Oncol 26: 2118-2123, 2008.

21. Tournigand C, Cervantes A, Figer A, et al: OPTIMOX-1: a randomized study of FOLFOX4 or FOLFOX7 with oxaliplatin in a stop-and-go fashion in advanced colorectal cancer - a GERCOR study. J Clin Oncol 24: 394-400, 2006.

22. Feliu J, Safont MJ, Salud A, et al: Capecitabine and bevacizumab as first-line treatment in elderly patients with metastatic colorectal cancer. Br J Cancer 102: 1468-1473, 2010. 